\title{
The trabecular bypass stent in a pseudophakic glaucoma patient: A I-year follow-up
}

\author{
Antonio M Fea \\ Monica Dogliani \\ Federica Machetta \\ Gabriella Lale-Lacroix \\ Beatrice Brogliatti \\ Federico M Grignolo \\ Istituto di Fisiopatologia Clinica, \\ Clinica Oculistica, Università di \\ Torino, Italy
}

\begin{abstract}
Purpose: To describe the 1-year success of a trabecular stent implant in a pseudophakic glaucoma patient. The stent is implanted through a small corneal incision under gonioscopic control. Intraocular pressure control was reported in cultured human anterior segment.

Design: Observational case report.

Methods: We report the case of a patient who was implanted and followed-up for 1 year at the Dipartimento di Fisiopatologia Clinica. The main outcome measures were intraocular pressure and visual field.

Results: The patient's intraocular pressure was controlled with topical beta-blockers for 6 months and without therapy for 6 months. Two diurnal curves demonstrated achievement of target pressure during the day. The 1-year visual field was unchanged.

Conclusions: We are not aware of previous reports of trabecular stent implantation in pseudophakic patients. The trabecular stent had minimal peri-operative morbidity. Identification of the implantation site is challenging and reduction of efficacy over time may occur.
\end{abstract}

Keywords: stent, trabecular bypass, intraocular pressure, glaucoma

\section{Introduction}

Glaucoma in pseudophakic eyes is not uncommon; both trabeculectomy and deep sclerectomy failure have been reported in the literature with higher rates in pseudophakic eyes than in unoperated eyes (Fontana et al 2006). The iStent (Glaukos Corp, Laguna Hills, CA, USA) is a heparin-coated titanium device that can be inserted ab interno in the trabecular meshwork under gonioscopic control, driving the aqueous into the Schlemm's canal; it can lower intraocular pressure (IOP) in cultured human anterior segments (Bahler et al 2004). The device inserted ab interno has shown to be clinically effective when used concomitantly with phacoemulsification (Feijoo et al 2006). We report a case of a pseudophakic glaucoma patient who achieved target pressure after the implant of the trabecular bypass stent.

\section{Case report}

A 68-year-old patient with primary open-angle glaucoma (POAG) underwent uncomplicated phacoemulsification (OD) and presented with elevated IOP in the operated eye 1 month postoperatively. Her prior medical history is as follows: renal colic with a diagnosis of nephrolitiasis, which was medically controlled and a previously reported intolerance to prostaglandin analogues. Additionally, the patient reported disinterest in further invasive surgery. See Table 1 for complete baseline information.

After the initial phaco surgery, her postop regimen consisted of steroids for 1 week, tapered over 2 weeks. At Day 10 follow-up, IOP in the operated eye was $19 \mathrm{~mm} \mathrm{Hg}$ (14 $\mathrm{mm} \mathrm{Hg}$ in the contralateral eye). Also at Day 10, the right visual field had a fascicular scotoma impending on the macular region, and a superior notch of the optic nerve head. By 1 month postoperation, her IOP had increased to $30 \mathrm{~mm} \mathrm{Hg} \mathrm{OD} \mathrm{(and} \mathrm{to} 15 \mathrm{~mm} \mathrm{Hg}$ 
Table I Clinical characteristics at baseline

\begin{tabular}{ll}
\hline Medical history & Asthma \\
& NIDDM \\
Previous surgery & Pace maker implantation (2000) \\
& LE cataract surgery (2003) \\
& OU: dorzolamide tid \\
Glaucoma therapy & RE: Brimonidine bid \\
& Acetazolamide $250 \mathrm{mg} 1 / 2$ tablet tid \\
& RE: $19 \mathrm{~mm} \mathrm{Hg}$ \\
IOP & LE: $10 \mathrm{~mm} \mathrm{Hg}$ \\
& RE: 535 \\
Central corneal thickness & LE: 543 \\
\hline
\end{tabular}

in the nonoperated eye). At that time, she was on dorzolamide TID in both eyes, with the addition of brimonidine in the right eye only twice daily. At this follow-up, there were no signs of inflammation and steroids were not felt responsible for the IOP rise because they had already been stopped and IOP was normal while she was on steroid treatment (ie, previous control at 10 days). She was followed every 2 weeks for an additional 2 months ( 3 months after initial phaco). Target pressure (IOP $<18 \mathrm{~mm} \mathrm{Hg}$ ) was achieved (IOP ranged from 12 to $17 \mathrm{~mm} \mathrm{Hg}$ ) with added medical therapy (acetazolamide $1 / 2250 \mathrm{mg}$ tablet TID). Following a significant eyelid and conjunctival allergic reaction, she was switched to preservative-free carteolol $2 \%$, brimonidine, and topical steroids. Inflammation returned after steroid use was discontinued.

To attempt better management of her glaucoma, her therapy was changed to preservative-free carteolol 2\% BID and acetazolamide $250 \mathrm{mg}$ TID. She was able to achieve target IOP of $<18 \mathrm{~mm} \mathrm{Hg}$ on maximum therapy, but did not want additional invasive surgery after an unsuccessful argon laser trabeculectomy was performed to avoid the use of systemic drugs.

Following a renal cholic, the patient agreed to the iStent implantation, as she did not view the procedure as invasive.

The device itself is a small L-shaped hollow tube designed to be implanted into Schlemm's canal by having the surgeon make a slit through the trabecular meshwork. The iStent is designed for nasal placement and is guided into Schlemm's canal using ab interno gonioscopy. In this patient, a nasal clear corneal $2 \mathrm{~mm}$ incision was made. The anterior chamber was then filled with a viscoelastic agent to reform the anterior chamber and provide more clearance in the angle. The surgeon (AMF) ensured the orientation of the stent on the applicator, placed the gonioscope on the cornea, and positioned the microscope to enable visualization of the trabecular meshwork. The anterior chamber was then traversed with the applicator. The leading edge of the iStent was eased through the meshwork and into the canal, with the tip directed inferiorly.

On the first postoperative day, her vision OD was maintained (25/20), few red cells were present in the anterior chamber, IOP was well controlled (12 $\mathrm{mm} \mathrm{Hg}$ ), and the stent was correctly placed in the angle (Figure 1). When pressure was applied to the goniolens, small blood reflux could be seen sideways from the stent and some blood was reabsorbed by the stent snorkel (video). Pressure was monitored monthly for 6 months and was always lower than $17 \mathrm{~mm} \mathrm{Hg}$ (range: $12-16 \mathrm{~mm} \mathrm{Hg}$ ) on carteolol; IOP remained $<17 \mathrm{~mm} \mathrm{Hg}$ during the day.

At 6 months, the stent remained in place and unobstructed (Figure 2, left); the UBM (Figure 2, right) demonstrated that the snorkel of the stent was adequately placed in the angle. Topical therapy was then discontinued OD and the patient followed for an additional 6 months (1 year after stent implantation). The IOP in the right eye remained $<18 \mathrm{~mm} \mathrm{Hg}$ (mean $14.9 \pm$ 1.7; range: $13-17 \mathrm{~mm} \mathrm{Hg}$ ). A diurnal curve without therapy demonstrated IOP $<17 \mathrm{~mm} \mathrm{Hg}$ (range: $12-16 \mathrm{~mm} \mathrm{Hg}$ ). The 1 -year visual field and CCT were unchanged.

\section{Discussion}

We are reporting on our own successful experience using a trabecular stent in a pseudophakic eye using an ab interno approach. Our experience mimics that of other surgeons using the stent in patients who were not pseudophakic (Spiegel et al 2007), suggesting the stent's efficacy in controlling glaucoma may not be limited to just virgin eyes. There is currently under way a larger clinical study of 47 patients with cataract and open-angle glaucoma to directly compare results in IOP lowering between those implanted with stents to those undergoing cataract surgery only and using medical therapy to manage their glaucoma.

Of note, in combined surgery, the ability for the surgeon to use the clear corneal incision created during cataract surgery can be advantageous, as there is no need for cautery and the conjunctiva and sclera are not disturbed. In this technique, a traditional glaucoma surgical procedures such as trabeculectomy would not be negatively affected (Samuelson 2007).

In this case study, the patient presented with a high IOP 1 month following uneventful cataract surgery and without any sign of inflammation. It is unlikely that steroids might have caused this IOP rise because they had already been tapered. The patient had a trabecular bypass stent 


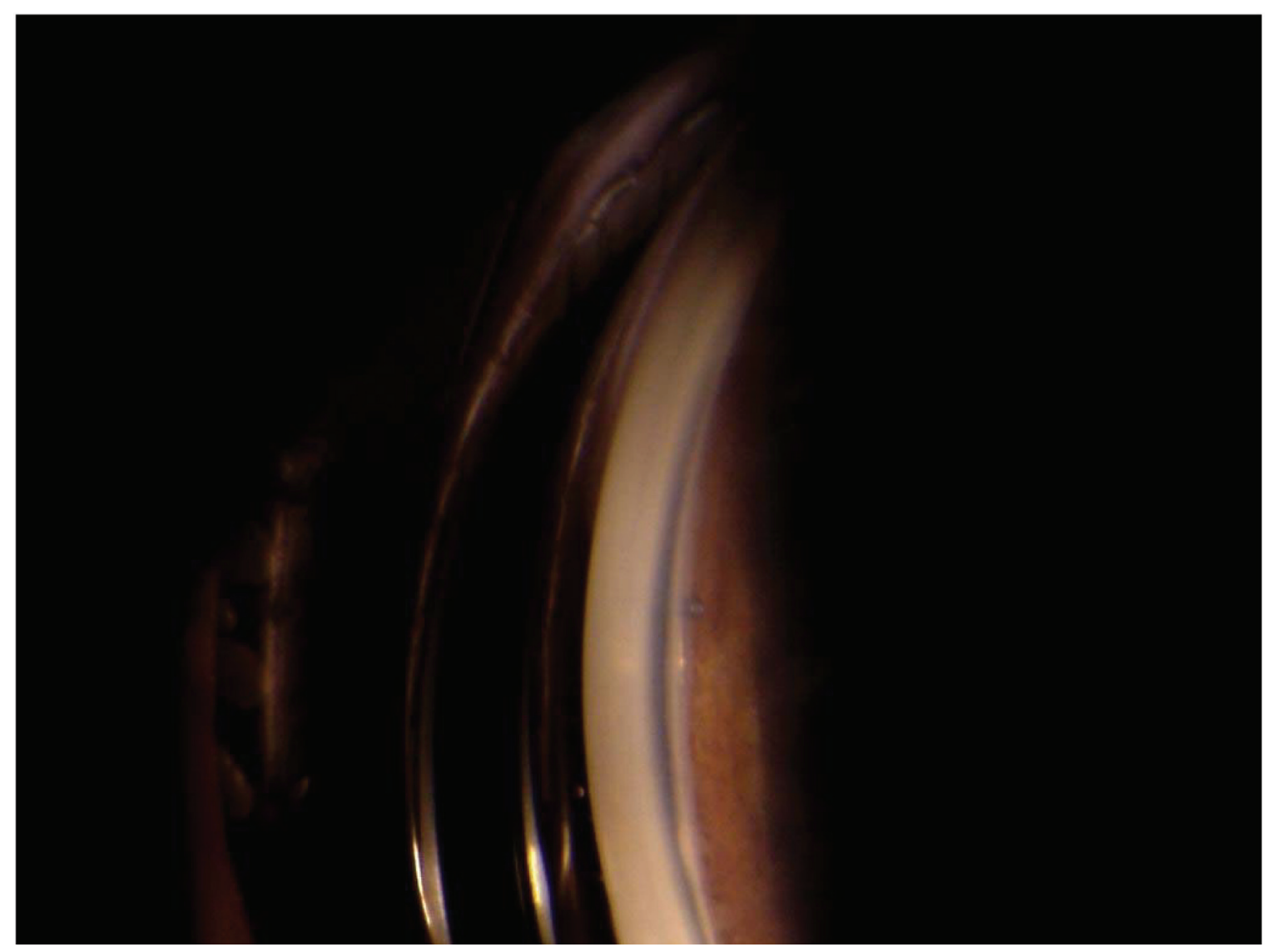

Figure I The stent is seen correctly placed at the trabecular meshwork level.

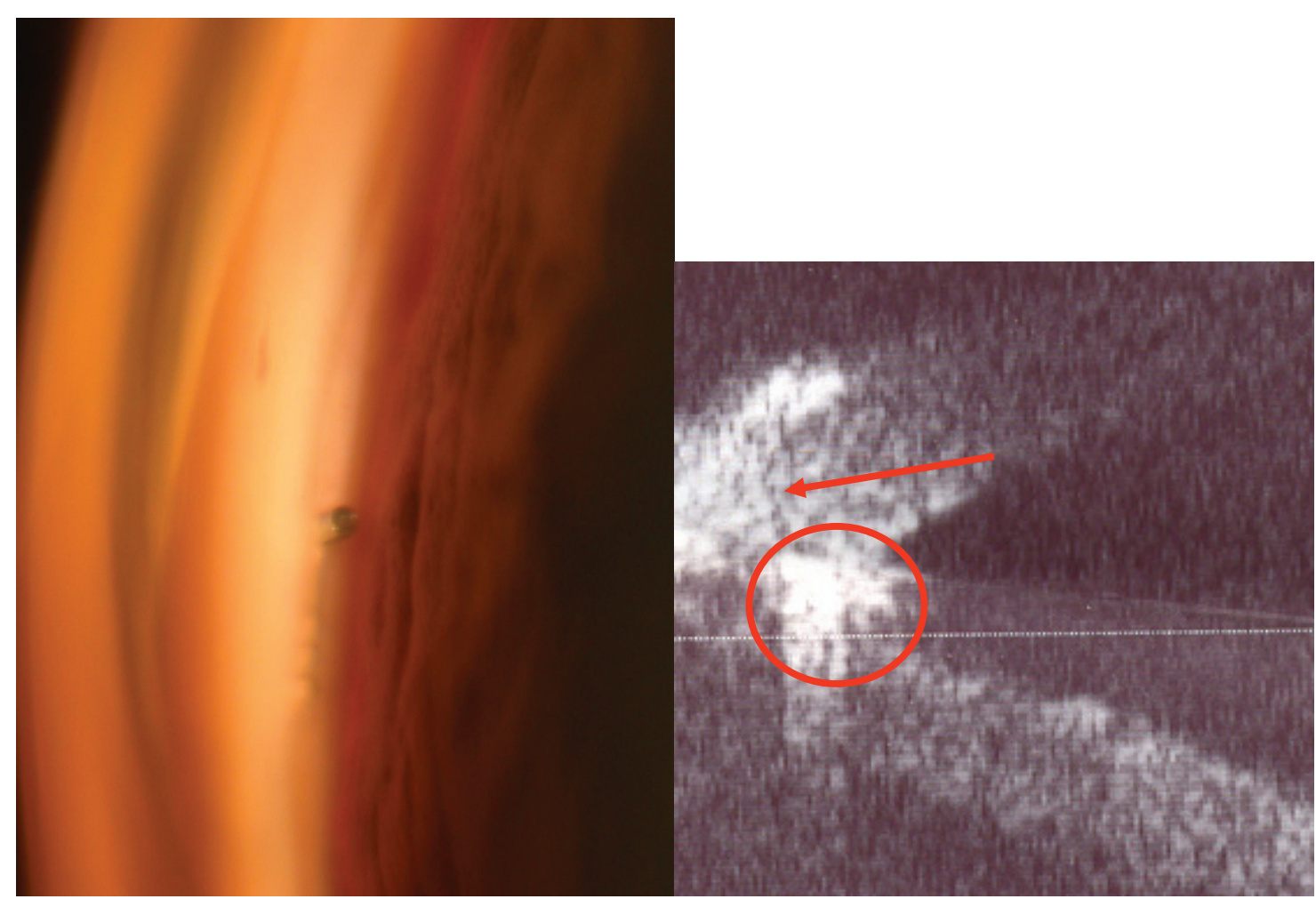

Figure 2 Left:The gonioscopic view of the stent. Right:The UBM shows the hyperechogenic structure of the stent (circle), placed below the corneal wedge (arrow). 
inserted through a $2 \mathrm{~mm}$ temporal corneal incision. The stent implantation was successful in maintaining IOP less than $17 \mathrm{~mm} \mathrm{Hg}$. A key to a successful surgery is the need of correct identification of Schlemm's canal and therefore proper placement of the stent. Although in enucleated eyes, the mean postoperative IOP averaged $12.4 \mathrm{~mm} \mathrm{Hg}$. In our patient, the mean IOP was slightly higher (14.9 mm Hg), possibly due to limited circumferential flow in Schlemm's canal. Additional stent placement might result in further IOP lowering as suggested by in vitro models (Bahler et al 2004). Longer-term IOP reduction has to be assessed with regard to continuous lumen clearance or possible future trabecular fibrosis.

\section{Disclosure}

This case report has never been presented at any conferences, although the video was presented as part of a longer one at the 2006 American Academy of Ophthalmology, Las Vegas, November 22-24, 2006. None of the authors have any financial interest in the product, procedure and material presented in the paper.

\section{References}

Bahler CK, Smedley GT, Zhou J, et al. 2004. Trabecular bypass stents decrease intraocular pressure in cultured human anterior segments. Am J Ophthalmol, 138:988-94.

Feijoo GJ, Garcia-Sanchez J, Spiegel D. 2006. Co-existing open-angle glaucoma and cataract: treatment by cataract surgery and the iStent Trabecular Bypass micro stent [Poster]. IGS meeting 2006, Tampa Bay, FL, USA, November 16-17, 2006.

Fontana H, Nouri-Mahdavi K, Caprioli J. 2006. Trabeculectomy with mitomycin $\mathrm{C}$ in pseudophakic patients with open-angle glaucoma: outcomes and risk factors for failure. Am J Ophthalmol, 141:652-9.

Samuelson TW. 2007. Trabecular bypass procedures gain ground in glaucoma surgery. Ocular Surgery News. August 15, 2007 [online]. Accessed Feb 5, 2008. URL: http://www.osnsupersite.com/view. asp?rID $=23159$.

Spiegel D, Wetzel W, Haffner DS, et al. 2007. Initial clinical experience with the trabecular micro-bypass stent in patients with glaucoma. $A d v$ Ther, 24:161-70. 